\title{
Del activismo docente, el saber pedagógico del maestro y su impacto en la realización profesional de sus estudiantes sordos
}

Teaching Activism, Teachers' Pedagogical Knowledge and their Impact on their Deaf Students' Career Success

Sobre o ativismo docente, os saberes pedagógicos do professor e seu impacto na realização profissional de seus alunos surdos

José Ignacio Bolaños-Motta* iD orcid.org/0000-0002-9976-1808 Elizabeth Casallas-Forero** (iD orcid.org/0000-0002-3590-5462 Viviana Jazmín Sabogal-Gámez*** (D) orcid.org/0000-0002-3293-5543

Para citar este artículo: Bolaños-Motta, J. I., Casallas-Forero, E. y Sabogal-Gámez, V. J. (2020). Del activismo docente, el saber pedagógico del maestro y su impacto en la realización profesional de sus estudiantes sordos. Revista Colombiana de Educación, 1(80), 153-172. https://doi.org/10.17227/rce.num80-10021

\section{(c) $(1) \Theta$}

* Magíster en Educación. Profesor de la Universidad de los llanos, Villavicencio, Colombia. Correo electrónico: jbolanos@unillanos.edu.co

** Magíster en Educación. Profesora de la Universidad de los llanos, Villavicencio, Colombia. Correo electrónico: ecasallas@unillanos.edu.co

*** Especialista en pedagogía y docencia. Profesora de la Universidad de los llanos, Villavicencio, Colombia. Correo electrónico: vsabogal@unillanos.edu.co 


\section{Resumen}

El presente estudio tuvo como objetivo exponer la existencia de una relación vinculante entre el éxito profesional de un grupo de diez docentes sordos de diversas ciudades del país, y las personas que fueron sus maestros durante su vida escolar y universitaria. Dentro del estudio se tomaron algunas consideraciones teóricas, respecto al tema del éxito profesional, además de algunas bibliografias referentes al tema de la discapacidad auditiva. Se siguió el método biográfico narrativo, para lo cual se usaron herramientas como la entrevista a profundidad y la cartografía social. En el apartado de los resultados se presentan dos categorías: 1) el activismo docente en los derechos de las personas en condición de discapacidad y 2) La importancia del saber pedagógico en el campo de la discapacidad. Como conclusión se da a conocer la existencia de una base para el buen desarrollo del educador del campo de la discapacidad, pues los maestros de las personas sordas que hicieron parte de este estudio contaban con excelentes cualidades humanas y profesionales.

\section{Palabras clave}

sordo; discapacidad; escuela; formación; profesionales de la educación

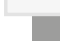

Keywords

deaf; disability; school;

training; education

professionals

\begin{abstract}
The purpose of this study was to expose the existence of a binding relationship between the career success of a group of ten deaf teachers from various cities in the country, and the people who were their teachers during their school and university years. Within the study, some theoretical considerations were taken, regarding the issue of professional success, as well as some bibliographies on the subject of hearing impairment. The studyfollowed a narrative biographical method, using tools such as in-depth interviews and social cartography. As a result, two categories emerged: (1) Teaching activism on the rights of people with disabilities, and (2) The importance of pedagogical knowledge in the field of disability. As a conclusion, the existence of a basis for the proper development of the educator in the field of disability is revealed, since the teachers of deaf people who were part of this study

had excellent human and professional qualities.
\end{abstract}

\section{Resumo}

O presente estudo teve como objetivo expor a existência de uma relação vinculativa entre o sucesso profissional de um grupo de dez professores surdos de várias cidades do país e as pessoas que foram seus professores durante a vida escolar e universitária. No âmbito do estudo, foram realizadas algumas considerações teóricas, a respeito da questão do sucesso profissional, bem como algumas bibliografias sobre o tema deficiência auditiva. Seguiu-se o método biográfico narrativo, para o qual foram utilizadas ferramentas como entrevistas em profundidade e cartografía social. Na seção de resultados, duas categorias são apresentadas 1) ativismo docente pelos direitos das pessoas com deficiência e 2) A importância do conhecimento pedagógico na área da deficiência. Conclui-se que existe uma base para o bom desenvolvimento do educador na área da deficiência, visto que os professores de surdos que fizeram parte deste estudo possuíam excelentes qualidades humanas e profissionais.

\section{Palavras-chave}

surdo; deficiência; escola; treinamento; profissionais da educação 


\section{Introducción}

Las tumbas de mis dos grandes maestros están aquí, he estado conectado a ellos durante muchos años, todo lo que recibo aún hoy, lo recibo a través de su alma.

Michael Laitman,

Sobre el deber ser del maestro - o el pedagogo- se ha dicho mucho a lo largo de la historia, de allí que exista toda suerte de virtudes vinculadas a dicha vocación. Uno de los propósitos que garantizan la calidad del maestro en su oficio (Saldarriaga, 2002) es atender la formación de ciudadanos, pretensión que varía no solo por periodos históricos, sino también por Estados nacionales. En virtud de lo anterior, ser educador implica una negociación entre el interés de la sociedad (o Estado), y el interés individual-vocacional de tal o cual docente. De tal modo, como parte de la formación ciudadana $-\mathrm{y}$ como un aspecto muy importante del modelo de sociedad que se proyecta-, es necesario examinar la realización profesional, que resulta ser tan multifactorial y compleja como la vida misma. Se asume, por tanto, que es susceptible de investigación explicar las causas por las cuales una persona o grupo humano —en este caso la población sorda-, Ilega o no a obtener éxito en su profesión.

En este sentido, el presente estudio pretende mostrar la existencia de una relación vinculante entre la realización profesional de un grupo de personas sordas y los docentes de colegios y universidades que se encargaron de brindar formación académica a dichas personas con discapacidad auditiva. Para ello, se trata de ahondar en los periodos de escolaridad —secundaria y universidad — de diez personas sordas, habitantes de las ciudades de Granada, Villavicencio, Restrepo y San José del Guaviare (en el departamento del Meta), y Tunja, capital del departamento de Boyacá. La totalidad de los actores de investigación se desempeñan como profesionales en diversas instituciones, entre las que se cuentan escuelas, fundaciones, microempresas, colegios y universidades. Todos concuerdan en que hubo en su vida un maestro que, en el ejercicio de su vocación profesoral, se encargó de fortalecer en ellos el deseo por el saber académico-profesional. Ahora bien, en los Estados modernos uno de los propósitos comunes (Laval y Dardot, 2013, 2015) es realizarse como profesional, cuestión que se logra después de haber cursado con éxito una carrera universitaria, lo cual, además, mejora el nivel de ingresos y garantiza la entrada al sistema productivo. ${ }^{1}$ Esto se encuentra directamente ligado a la concepción

1 Hablar de profesionalidad y sistema productivo es en sí mismo un tema debatible. No obstante, los sordos sí se preocupan por ello pues no desean depender de nadie, sino ser profesionales y ganar dinero. 
moderna de una "educación para el trabajo" (Messina, Pieck y Castañeda, 2008, p. 24), lo cual resulta ser muy cuestionado — con justas razones_ por sectores muy importantes de las humanidades, pues el ideal del "éxito" en la sociedad moderna se relaciona con el imperativo neoliberal que alude a una "optimización personal [que] sirve únicamente para el funcionamiento perfecto dentro del sistema" (Han, 2014, p. 42), y es dentro de este sistema que el sujeto de rendimiento se pretende a sí mismo como libre, aunque, como lo menciona este mismo autor

Es en realidad un esclavo. Es un esclavo absoluto, en la medida en que sin amo alguno se explota a sí mismo de forma voluntaria. No tiene frente a sí un amo que lo obligue a trabajar. El sujeto del rendimiento absolutiza la mera vida y trabaja. La mera vida y el trabajo son las caras de la misma moneda. (Han, 2014, p. 7. Énfasis en el original)

No obstante, en esta investigación no se hace referencia a la idea de éxito profesional desde la óptica del lucro económico y la explotación, sino más bien desde una perspectiva de realización profesional de un grupo de personas, cuyo objetivo es prestar un servicio educativo o social a diversos sectores, que de una u otra forma atienden a poblaciones relacionadas con la discapacidad. Así las cosas, se revisaron estudios precedentes en torno al éxito profesional (Martínez et al., 2012; Mercé, 2012) y con relación a temáticas como la inteligencia emocional (De Haro y Castejón, 2014), el acceso a la educación superior (Rodríguez et al., 2008), los procesos de escolarización universitaria de personas sordas (Santos et al., 2008), y el éxito profesional en campos como el fútbol (Castellano, 2018) y las ciencias jurídicas (Águila, 2010). A partir de lo anterior, se consideró necesario llevar a cabo un estudio de esta naturaleza, esta vez, dando una mirada a profesionales en condición de discapacidad.

Para el caso que nos ocupa, como ya se mencionó, se abordó el tema del éxito o realización profesional partiendo de la experiencia de personas con discapacidad auditiva, quienes no escapan a las bondades y problemáticas del sistema productivo y sus exigencias. Dicho sistema, bien sabemos, lleva a que cualquier persona deba sortear con éxito decisiones como optar por adelantar estudios profesionales y culminarlos, cursar un posgrado en su disciplina y tener una estabilidad laboral que le permita vivir dignamente. Pues bien, todas las metas y decisiones expuestas son mucho más difíciles de alcanzar para una persona sorda, pues estudios pretéritos exponen que: "en Colombia sólo el diez por ciento (10 \%) del total de las jóvenes y los jóvenes con discapacidad logran ser bachilleres, y de ellos sólo el cuatro por ciento (4 \%) alcanza niveles de educación superior" (Fernández y Vasco, 2012, p. 473). 
En esa dirección, el diálogo con los actores de investigación ${ }^{2}$ dio a conocer algunas circunstancias por las que atraviesa la población en situación de discapacidad, pues múltiples personas de la sociedad, en diferentes contextos educativos, Ilegaron a referirse a ellos con palabras de desprecio como: "tú sordo, mejor tú ser zapatero", "tú sordo, tú brillador de zapatos", "tú sordo, tú vendedor de dulces", "ustedes todos hagan manualidades", "ustedes todos sordos hacer pan mejor, no venir a universidad" (Edison, comunicación personal, abril del 2018). Sentencias como estas implicaron que ellos se subvalorasen con pensamientos como los siguientes:

Yo pensar, yo mejor hacer pan, porque profesora mujer decir que en universidad yo fastidio.

Yo mejor no ir universidad, yo no gustar, yo mejor trabajar en taller-madera... profesores odiar discapacidad.

Yo mejor matarme, yo no servir para mundo, yo no futuro. (Ferrigno y Goya, comunicaciones personales, marzo y junio del 2018) ${ }^{3}$

Con lo anterior, vemos la presencia de determinados estereotipos sociales (Adajus, 2013) sobre el ser sordo, a partir de los cuales se cree que sus vidas "están condenadas a una vida sin esperanza" (Ferreira, 2010, p. 62); en muchos casos esto conduce a que la mayoría de personas en situación de discapacidad renuncie a estamentos educativos (bien sea en secundaria o en Universidad). Las acciones anteriores son además producto de una cultura que se ha naturalizado como benefactora para unos: la "sociedad oyente" (Llorent y López, 2010), y excluyente para otros: la minoría sorda (Pérez, 2014). No obstante, en aquellos momentos en que personas e instituciones ${ }^{4}$ hacen parte de las realidades difíciles para la población con discapacidad auditiva, aparece en la vida de los sordos un maestro que se encarga de sopesar los problemas desencadenados por aquellos estereotipos sociales, y ayuda a cultivar la posibilidad de generar un futuro profesional, cultivando aspectos como: el amor por el saber académico, la comprensión y la ayuda a quien nadie está interesado en ayudar, el conocimiento de sus derechos como ciudadano y como

2 Dentro de este artículo no se proporcionan los nombres reales de los actores de investigación, por lo tanto, estos fueron reemplazados por los de diversas celebridades del mundo de las artes escénicas y de la música (entre otros, como Lou Ferrigno, Halle Berry, Thomas Edison, Ludwig van Beethoven, Jodie Foster, Whoopi Goldberg, Francisco Goya, Luis Miguel, Ozzy Osbourne y Eric Clapton. Algunos de ellos nacieron sordos, otros perdieron la audición con el paso de los años.

3 Las referencias a los actores de investigación fueron traducidas de la Lengua de Señas Colombiana a la Lengua Castellana. Se intentó guardar fidelidad sobre los testimonios, por cuanto al tratarse de personas sordas no se usan conectores.

4 En términos del presente estudio, personas e instituciones parecieran no estar tan diferenciadas en sus opiniones, pues juntas son igualmente excluyentes. 
persona en condición de discapacidad y el valor para enfrentarse, a través del conocimiento de los derechos constitucionales, a las constantes exclusiones que vive la población sorda.

Ante este panorama educativo y también social, en la mayoría de los casos desolador, los investigadores encargados de la presente propuesta se dieron a la tarea de vincular como objeto de estudio las experiencias y vivencias (Dilthey, 2015) por un grupo de docentes que, en su momento, "bien actuaron" al atender la formación de las personas en el campo de la discapacidad.

En un primer momento surgió la siguiente pregunta de investigación: ¿Qué factores resultaron determinantes en el éxito profesional de un grupo de diez personas sordas que hoy en día se desempeñan en el campo de la educación? No obstante, luego de las primeras entrevistas se logró determinar un factor general que había influenciado positivamente la realización profesional de los actores sordos: la presencia de docentes que permitieron y ayudaron a sortear toda clase de problemas y a que culminaran sus estudios, además de que se interesaran por continuar con una vida profesional, de allí que la pregunta que finalmente direccionó la investigación fue: ¿Qué características tenían los docentes de diez estudiantes sordos, que relacionan su éxito profesional con la formación académica que recibieron por parte de sus maestros? Desde ese cuestionamiento, entonces, se buscó evidenciar la importancia del rol del maestro como ente humanizador de influencia sobre las instituciones educativas, y como potencializador de las posibilidades de ser (Foucault, 2003) del individuo con una discapacidad auditiva.

\section{Metodología de investigación}

Esta investigación se realizó usando el método inductivo (Pasek, 2008), ya que se propuso desarrollar una interpretación de la realidad social (Álvarez y Jurgenson, 2009) de un grupo de profesionales sordos que han tenido cierto grado de éxito en sus profesiones. En este orden de ideas, se llevó a cabo una investigación desde el enfoque cualitativo (Pasek, 2008), dada la posibilidad de describir la realidad de un grupo humano inscrito dentro del campo de la discapacidad auditiva. En concordancia con lo anterior, se pretendió que de forma espontánea cada uno de los actores sociales (exestudiantes sordos que hoy en día son maestros en ejercicio), contaran sus historias de vida (González, 2019; Huchim y Reyes, 2013), indicando de manera muy puntual, sus vivencias dentro de diversas instituciones educativas, en Villavicencio (departamento del Meta).

Así, con la intención de lograr una visualización de las problemáticas sociales que han acaecido durante los diversos momentos del ciclo de vida (Fayad, 2015) de cada uno de ellos, se realizó un grupo de conversaciones 
y entrevistas, en diversos lugares, aunque es necesario decir que la mayoría de los encuentros se realizaron de forma virtual. ${ }^{5}$ En principio, la investigación biográfico-narrativa pretendió indagar acerca de la forma como este grupo de sordos logró convertirse en profesional, y además ejercer su profesión con cierto grado de influencia sobre su entorno social. De tal modo, la investigación biográfico-narrativa se constituyó como:

un lugar de encuentro e intersección entre diversas áreas sociales, que relaciona diversos saberes, como la teoría lingüística, historia oral e historia de vida, la antropología narrativa y la psicología [...] permite dar significado y comprender los aspectos cognitivos, afectivos y de acción de los docentes, esto se debe a que ellos, cuentan sus propias vivencias. (Huchim y Reyes, 2013, p. 2)

En atención a lo anterior, la recolección de datos se realizó a partir del uso de entrevistas a profundidad. Esta fue la herramienta que más importancia tuvo en este proceso investigativo, ya que este ejercicio permite observar en detalle "la vida del otro, penetrar y detallar en lo trascendente, descifrar y comprender los gustos, los miedos, las satisfacciones [...] relevantes del entrevistado; consiste en construir paso a paso y minuciosamente la experiencia del otro" (Robles, 2011, p. 40). El proceso de entrevista se realizó con el objetivo de indagar, a través de diversas conversaciones, las características que tuvo la vida (en general y en el campo profesional), de cada uno de estos actores de investigación. Con ello se buscó detectar aspectos relacionados con modelos educativos, maestros, escolaridades y demás elementos que de una u otra forma dieron señales a la "rosa de los vientos" para que los sordos tomasen decisiones sobre su futuro, pese a las condiciones de un país que no suele ser garante de los derechos sociales de la población denominada legalmente discapacitada. Además de lo anterior, se realizó un ejercicio de cartografía social, entendiendo que se trata de una estrategia a través de la cual se indaga a "un determinado número de personas que tienen como perspectiva alcanzar un objetivo común vinculado con el conocimiento" (Carballeda et al., 2012, p. 3; Vélez et al., 2012). ${ }^{6}$ Las cartografías sociales se desarrollaron después de la entrevista y a través de ellas se pretendió que los actores de investigación plasmaran sobre el papel diversidad de dibujos relacionados con las características generales y distintivas de los maestros que acompañaron, en determinado estadio de la vida, sus procesos de formación académica. ${ }^{7}$ En este mismo

5 Algunas entrevistas se realizaron en los hogares paternos de los actores de investigación, la mayoría de ellas se hicieron por videollamada en redes sociales como Facebook y WhatsApp.

6 Aunque este modelo de investigación ha tomado mucha fuerza, hasta llegar a convertirse en sí mismo en una metodología de investigación, en este estudió se usó como una estrategia de recolección de la información.

7 Durante el ejercicio se solicitó a los actores de investigación que, en la parte de atrás de sus dibujos, plasmaran en lengua castellana, el significado de sus gráficos.

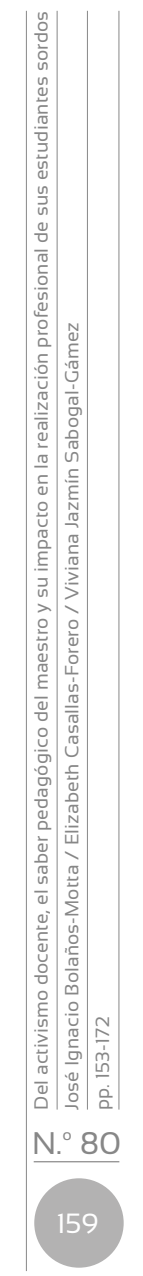


orden de ideas, la cartografía social resultó de gran ayuda para el proceso investigativo, dado que esta experiencia se configuró como "una estrategia de recolección de la información destinada a graficar la construcción de las problemáticas o significaciones de vida" (Bolaños, 2019, p. 55) de los profesionales sordos. Si bien esta actividad fue compleja para ellos, fue tan valioso como las mismas entrevistas, ya que, gracias a estos gráficos se logró indicar la importancia de los maestros en sus vidas. Finalmente, en el intento de hacer una interpretación de los relatos hechos por los actores de investigación, se desarrolló un trabajo hermenéutico ${ }^{8}$ sobre las cartografías y los relatos de los actores de investigación. Entonces, se asumió como criterio de calidad la coherencia entre los datos recogidos, lo que dio lugar a "nuevas ideas y maneras de ver las cosas y las que se han estudiado" (Flick, 2015, p. 92), todo lo anterior a partir del cruzamiento de datos entre las diversas estrategias de recolección.

\section{Resultados}

A partir del proceso investigativo realizado a lo largo del año 2018 y parte del 2019, se logró como resultado desarrollar las dos categorías centrales del presente texto, se hace referencia a dos capacidades básicas para el maestro que ejerce su saber-hacer en el campo de la discapacidad: activismo docente en los derechos de las personas en situación de discapacidad, y la importancia del saber pedagógico en el campo de la discapacidad. Categorías que serán expuestas de manera discriminada a continuación.

\section{El activismo docente en los derechos de las personas en situación de discapacidad}

Una de las características observadas en la mayoría de los profesionales con quienes se realizó la presente investigación es el hecho de que muchos de ellos, quizá todos, contaron con una buena escolaridad, ya que sus condiciones académico-profesionales estuvieron determinadas en buena medida por las instituciones universitarias en las cuales formaron su ser y su ser profesional. Si bien para muchas personas resulta claro que las academias universitarias se deben básicamente a sus maestros y a la calidad de estos, en el caso de la población sorda las cualidades $O$ calidades del docente universitario sí que resultan ser determinantes para el futuro del profesional sordo. La academia universitaria es entonces aquel lugar donde se premia el desempeño intelectual de las personas; se observa que algunos formadores universitarios se encargaron de incentivar 
un activismo social en la población sorda de la que se ocuparon durante su formación académica, de cara a atender sus necesidades educativas. Aunque quizás plagado de tropiezos de todo tipo, es el docente universitario quien a través de un discurso académico anclado a diversas áreas de las humanidades abre campo a un primer acto emancipador, en el entendido de que "con la palabra y el acto nos insertamos en el mundo humano" (Arendt, 2001, p. 24). Desde esa perspectiva, es posible para el docente identificar las inequidades hacia la población sorda y tejer las propuestas que pretenden solventar en parte las necesidades educativas de las personas con esta discapacidad, con el fin de "hacerse cargo" de quienes no se encuentran en las mismas condiciones, básicamente comunicativas, para recibir educación universitaria a través de la expresión oral. Dichas formas emancipadoras infundidas en algunas de las personas sordas se ven reflejadas en los actos legales que tuvieron que llevar a cabo para acceder a la educación y hacer cumplir su derecho a estudiar. Específicamente nos referimos a la tutela como el mecanismo que resulta determinante en la defensa de los derechos de las personas con discapacidad auditiva; de hecho, tal como narran los actores sociales de esta investigación, fue necesario para ellos hacer uso de esa herramienta legal:

En el año 2002 yo hice tutela Universidad, profesora ayudarme porque yo quiero estudiar, dijeron no para yo educación superior. Yo puntaje bajo Icfes, yo malo para la Lengua Castellana, lengua castellana yo segunda lengua. Siete meses derechos de petición más acción de tutela, proceso largo y yo ganar acción de tutela y empezar estudiar. (Berry, comunicación personal, octubre del 2018)

Universidad dice difícil no saber cómo sordos, intérprete no hay, tocó tutela, ellos no quiere, pero nosotros sordos derecho educación pelear, yo ganar tutela ellos aceptar [posteriormente...] yo universidad siempre esfuerzo y bien beca siempre ganar. (Ferrigno)

Universidad difícil, pero yo apoyo con amiga acción de tutela ganar, sordos rechazados, yo soy malo para el resultado Icfes, yo Icfes bajo, y tampoco inclusión, yo entrar estudiar en la carrera profesional para Licenciatura, Universidad darme servicio de intérprete de Lengua de Señas Colombiana-español. (Edison, comunicación personal, abril del 2018)

Lo cierto es que las dificultades para esta población se presentan incluso desde la educación secundaria, pues:

Después entrada de un colegio para bachillerato es muy difícil, gracias profe Mariano que hizo una demanda a la Secretaría de Educación Distrito por derecho en la educación y la igualdad por oportunidad. Se logró que un colegio se Ilama Distrital Jorge Eliécer Gaitán se aceptó la integración escolar para las personas sordas desde 6 hasta 11. (Beethoven, comunicación personal, enero del 2019) 
[...] Pero otros docentes no comprende inclusión ni pensamiento de persona sorda, entonces algunos bravos por sordo en el salón, entonces colegio dice sordos mejor en la casa después venir cuando colegio tenga intérprete, esta difícil y esperar. (Foster, comunicación personal, mayo del 2018)

De lo anterior se infiere que aunque existen marcos legales que permiten hacer cumplir los derechos de la población sorda, en muchas ocasiones las instituciones educativas, y en ellas los respectivos docentes, suelen rechazar las posibilidades de impartir educación a las poblaciones con discapacidad, ${ }^{9}$ lo cual, en muchos casos, se debe a la ausencia de una diferenciación conceptual entre "enfermedad y diversidad funcional" (Palacios y Romañach, 2008, p. 117). En vista de la resistencia generada por diversas entidades de la educación secundaria y universitaria, muchos sordos del departamento del Meta interponen acciones de tutela como mecanismo para hacer valer sus derechos constitucionales. Pero, de acuerdo a los testimonios de los actores sociales de esta investigación, las acciones legales que interpusieron en determinados momentos fueron previamente impulsadas por aquel docente universitario que al observar determinada injusticia tomó parte como activista de los derechos de las personas sordas. El activismo, vale aclarar, es un movimiento de

Índole política pues en su esencia conduce a la educación ideológica de los ciudadanos; por ello es ampliamente utilizado en función del trabajo con las masas y asume diferentes formas para defender proyectos, enfrentar realidades, combatir situaciones [y en esta materia] estrategia importante para lograr la participación en los cambios sociales y el desarrollo. (Reyes y Colás, 2017, pp. 41-42)

En muchos casos, los maestros que se encargaron de dar la lucha jurídica por la garantía de los derechos de los estudiantes sordos también estuvieron atentos a los procesos de entrada y sostenimiento en los centros educativos. Lo anterior se resume en que los maestros debieron atender al mismo tiempo procesos judiciales, administrativos y académicos, lo cual en sí mismo demanda mucha energía. Las acciones de tutela en su momento fueron una medida final y forzosa ante la imposibilidad de llegar a una solución concertada con las instituciones educativas, y fue gracias a ella que determinados derechos fueron concedidos por medio

9 En ciertas universidades a algunos sordos se les otorga un intérprete, quien se encarga de reproducir el discurso del docente. No obstante, la adquisición de este talento humano como ayuda comunicativa suele ser un logro final, producto de una lucha legal por la reivindicación de un derecho inclusivo. 
de un acto jurídico. ${ }^{10} \mathrm{El}$ hecho de que los maestros se encargasen de las diversas necesidades de la población sorda a la cual atendieron, se observa en testimonios como los siguientes:

Profe hizo acción de tutela para Universidad, ellos aceptar solución para yo entrar estudiar en la carrera profesional Licenciatura.

Amiga profe acción de tutela, sordos rechazados resultado de Icfes puntaje muy bajo.

Tocó tutela, ellos no quiere, pero nosotros sordos derecho educación pelear, ganó tutela ellos aceptación por fin. (Goldberg y Beethoven, comunicación personal, abril del 2018)

Por esta razón, los maestros, tras encontrar cierto número de problemáticas, desarrollaron toda suerte de técnicas escriturales para entablar derechos de petición, tutelas y demandas judiciales. Además, aprendieron a relacionarse con los abogados y, por ende, a sortear diferentes clases de problemas con relación a la educación de las personas sordas, tal como lo manifiesta otro testimonio: "conseguir abogado, poner para proceso universidad darme estudio, luego ya conocer para problemas" (Goya en cartografía social, junio del 2019). Aunque es posible que los maestros hayan adquirido esta clase de saberes en sus carreras profesionales, se cree que la mayor parte de este conocimiento lo aprendieron sobre la marcha al buscar solución a los problemas de sus estudiantes.

Se diría, entonces, que existe una paradoja en lo referente a la relación población sorda-instituciones educativas, pues son estas últimas las que "se hacen las sordas"11 — valga la coloquialidad de la expresión—, con respecto a las problemáticas que aquejan a la población sorda. Con todo lo expuesto se puede concluir que los derechos de la población en condición de discapacidad no son en sí mismos una práctica social, sino una lucha social en la que el maestro cobra un gran protagonismo como conocedor de las instituciones, de la legalidad vigente y como activista de los derechos de algunos de sus estudiantes.

Los docentes de las personas sordas relacionadas en esta investigación hicieron activismo a través de acciones relacionadas con la exigencia de los derechos; no obstante, hoy en día sus estudiantes se desempeñan con éxito en oficios quizás más complejos. Quizás, los buenos estudiantes sí superan a sus maestros. Al margen de los actos judiciales a los que ellos también se ven expuestos, los profesionales sordos de hoy han alcanzado sus propios

10 A pesar de ello existen luchas y reivindicaciones por desarrollar, en algunas instituciones educativas se maneja por normatividad el otorgar cupo solo a un sordo por carrera. Al parecer, las causas de esta medida son de tipo económico.

11 Esta expresión coloquial colombiana se usa para indicar que una persona no escucha a otra, a causa de un disenso generado por la diferencia de opiniones. 
modos de realización profesional a través de nuevas formas de activismo como son: trabajo en fundaciones, dirección de noticieros regionales (para sordos), cargos directivos en colegios, y desde la docencia universitaria, entre otras actividades de no menor valía para la sociedad sorda.

\section{Importancia del saber pedagógico en el campo de la discapacidad}

Ya desde finales del siglo anterior Olga Lucía Zuluaga (1999) venía afirmando que la pedagogía hace referencia a una ciencia que "conceptualiza, aplica y experimenta los conocimientos referentes a la enseñanza de los saberes específicos, en las diferentes culturas" (p. 11). A partir de ello, se constituyó la idea del denominado movimiento pedagógico (Unda y Gutiérrez, 2015, p. 8) y en él, la noción de saber pedagógico como "un concepto metodológico central [que de una u otra forma] expresa los discursos sobre la enseñanza y la educación" (Ortiz, 2017, p. 183). En este sentido, un maestro ha de contar con la habilidad para conceptualizar, reflexionar sobre su práctica y aplicar su saber-hacer hacia la población sorda, en la noble búsqueda de disminuir —o idealmente desaparecer-, la brecha entre sordos y oyentes, como parte de la fortaleza que se deriva de la unión entre el "Discurso Pedagógico y el sujeto pedagógico" (Bernstein y Díaz, 1985, p. 3). Ello por cuanto la pedagogía es la ciencia que se encarga de llevar al ser humano desde su ser original hasta su cultura. ${ }^{12}$ Ahora bien, es importante tener en cuenta que los modelos educativos direccionados a la atención a la discapacidad auditiva, quizás debido al escaso avance en torno al concepto de pedagogía en siglos pasados, estuvieron por mucho tiempo en manos de las ciencias médicas; como consecuencia, "la medicina desplazó a la pedagogía" (Burad, 2010, p. 2). Por lo tanto, son los investigadores del campo de la pedagogía quienes deben ahora recuperar este espacio de acción, de tal suerte que la pedagogía logre establecer posibles transversalizaciones entre las disciplinas de la salud y de la educación. ${ }^{13}$

Estas transversalizaciones, quizás se han logrado en pocos casos, por cuanto algunos maestros, en este estudio quienes participaron en la formación académica del grupo de diez personas sordas, han tenido un compromiso social y político que ha impactado directamente en el trabajo de aula. Esta influencia y compromiso pueden entenderse específicamente en la medida en que se indaga sobre la razón por la

12 La cita inicial de Zuluaga cobra valor, ya que los sordos deberían ser vistos como parte de una cultura en particular pues poseen sociedades propias, artes propias, economías propias y lenguaje propio.

13 La tensión expuesta acude a la diferencia de enfoques, por un lado el biológico y por el otro "el enfoque social de la discapacidad" (García, 2005, p. 246). 
cual los maestros se comprometen y asumen la formación académica y el activismo para esta población con discapacidad auditiva. Lo que puede encontrarse, por ejemplo, es que si bien en términos generales el maestro de escuela o universidad colombiano rara vez conoce la lengua de señas colombiana, si por casualidad llega a contar con este saber, es porque muy seguramente se encuentra ante una determinada necesidad familiar, en la que por fuerza del destino, aprendió dicho saber-hacer. Esto no debería ser así, pero en general sí se aprende por necesidad. No obstante, sean cuales fueren las razones que motivan a un docente oyente a aprender la lengua de señas colombiana, dicho docente resulta ser altamente valioso para los procesos de los sordos en la escuela colombiana.

La única profesora oyente sabe poco Lengua de Señas Colombiana y me enseño Lengua de Señas Colombiana en materias colegio, ella única hablarme. (Miguel, comunicación personal, mayo del 2019)

En primaria, allí profesores trabaja solo oyentes pero ellos aprenden Lengua de Señas Colombiana, ellos trabajar modelos lingüísticos, yo y sordos mejorar aprender comunicación [en] Lengua de Señas Colombiana. (Osbourne, comunicación personal, marzo del 2018)

Si bien se sabe que "la familia [es] considerada como el contexto básico de desarrollo humano" (Henao y García, 2009, p. 787) y de ella dependen tanto el desarrollo lingüístico como profesional de la persona, es necesario crear transformaciones que motiven a la escuela a velar por la defensa de los derechos de las minorías. En este punto es el Estado, precisamente, quien habrá de velar por la creación de posgrados, seminarios, instituciones o fundaciones encaminadas a fortalecer competencias en los docentes de las escuelas y colegios. De esta forma, la persona se verá en la posibilidad de cultivar este saber, de la misma manera como se cultivan otros saberes académicos.

Con todo lo expuesto, vemos entonces que una de las causas del éxito profesional de muchos de los sordos consultados en la presente investigación es el hecho de que la mayoría de ellos contó con buenos maestros; docentes que ostentaban una sólida formación académica y que además se comunicaban en lengua de señas colombiana. Evidentemente, gracias a estos dos saberes lograron abrir las posibilidades de ser del sujeto (Foucault, 2003) y del futuro de sus estimados estudiantes sordos. De esta manera puede decirse que, en muchos casos, se venció el estigma social (Goffman, 2006) de que el sordo solo puede desempeñarse en oficios por fuera de la profesionalización universitaria.

De acuerdo a lo anterior, a partir de los datos obtenidos en las entrevistas y las cartografías realizadas a dichos profesionales sordos se observó que los docentes habían logrado fortalecer diversos saberes, como la

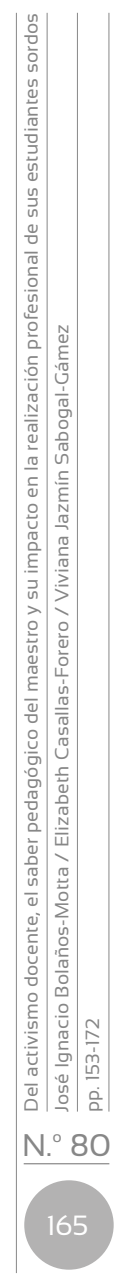


lengua de señas colombiana, la recurrencia a la corporeidad como forma de enseñanza y diversas competencias en didácticas de aula, como se evidencia a continuación:

En la sede sordos, mucho mejor, yo ser niño desarrollar pensamiento y lengua en señas con contacto persona sorda aprendido la lengua de señas es canal natural. (Clapton, cartografía social, febrero del 2019)

Profesora enseñarme siempre usar oral en las materiales pero ella sabe simple facial y corporal. (Beethoven, comunicación personal, enero del 2019)

Y es en el saber pedagógico donde se ven mezcladas diversas disciplinas y métodos de enseñanza, como es caso de la logogenia, la cual, unida a un compromiso social, logra impactar positivamente el saber-hacer en el aula:

Gracias proyecto profesora, ella hacer enseñanza a sordos, yo aprender español, metodología logogenia yo aprendí mejor a leer y escribir. (Berry, comunicación personal, octubre 2018)

[...] aprendido mucho, profesores colegio exigir mucho duro por espacio académico, todas áreas mejorar habilidades conocimiento, ellos aconsejaron futuro nosotros poder llegar ser profesional, profesora muy responsable, mucho compromiso por cambio paradigma sociedad. (Ferrigno, comunicación personal, marzo del 2018)

Muchos de los maestros que con gran importancia se encargaron de desarrollar las potencialidades o las inteligencias (Agurto, 2007; Gardner, 2001 ; 2016) de los actores de investigación aquí mencionados, contaron con espacios de la escolaridad universitaria para apoyar a sus futuros estudiantes. Ello se evidencia en expresiones como las siguientes:

Los profesores universitarios muy buenos, mi carrera es ciencias sociales, es muy excelente y mente abierta por reconocimiento de persona sorda, yo exigir espacio académico universitario y yo manejo discurso académico de LSC. (Beethoven, comunicación personal, abril del 2018)

En la Universidad yo mucho estudiar y practicar teoría libros ello sirve mucho, yo aprender muy bien, ahora yo trabajo ser muy bien. Yo docente universitario. En institución educativa administración muy contenta conmigo, y comunidad dice mi trabajo muy bien. (Goya, comunicación personal, junio del 2018)

Ahora bien, a diferencia de muchas poblaciones sordas de otras localidades del país, la población sorda de Villavicencio cuenta con la Asociación de Sordos del Meta (Asormeta), gracias la cual han podido tener maestros con buena formación en la lengua de señas colombiana. A lo anterior se agrega la posibilidad de generar múltiples relaciones entre pares y de verse como cercanos a una "Cultura sorda" (López-González y Llorent, 2013, p. 1670), 
cuestión que difiere de lugares como entornos barriales u otros espacios, en donde la exclusión y la desigualdad se hacen más comunes, ante las percepciones de inferioridad (Oliver, 1996) en el aspecto social-comunicativo. No obstante, al margen de la Asociación, los procesos de relacionabilidad de las personas sordas resultan ser mucho más complejas, particularmente en la etapa de escolaridad, aunque las dificultades no solo son para las persona sordas, sino también para sus docentes. ${ }^{14}$ Esto da lugar a múltiples disensos en el entorno educativo, de allí la importancia que cobran aquellos docentes que, como se vio en los casos aquí descritos, tuvieron, además de las cualidades académicas y personales, el interés y el compromiso de aprender para ayudar a formar profesionales cuya condición de discapacidad auditiva ha hecho difícil, pero no imposible, su andar en el entorno educativo.

Lo cierto es que si un docente colombiano desea generar una transformación de su comunidad educativa de cara a la atención de sus estudiantes sordos, ha de contar con la lengua de señas colombiana dentro de su saber pedagógico. De no ser así, su accionar pedagógico se verá muy limitado. Dominar la lengua de señas, de cualquier país, es un pre-saber para acercarse al estudiante con discapacidad auditiva. La comunicación asertiva no ha de habitar únicamente en manos de un intérprete. En mérito de lo anterior, la virtud de los docentes rememorados en esta investigación está en poseer como parte de su saber pedagógico una sólida disciplina de estudio, sumada a la capacidad de comunicarla asertivamente.

Es de destacar que, hoy en día, sus estudiantes perciben que, en buena medida, su éxito profesional proviene de actividades pedagógicas que hoy en día ellos realizan, algunas están relacionadas con la docencia directa, otras con el trabajo con comunidades. Lo anterior, entendiendo que la pedagogía se encuentra presente en la mayoría de los actos comunicativos del ser humano.

\section{Conclusiones}

Como se pudo observar en los apartes y categorías anteriores, ${ }^{15}$ ejercer la labor docente en el campo de la discapacidad auditiva implica, por un lado, que el maestro sea un activista de los derechos de las personas en

14 Dados los problemas de comunicación de la persona sorda, esta tiende a ser más conflictiva que los demás estudiantes y ello se manifiesta desde la escuela hasta la universidad. Esto se debe a que es precisamente en estos lugares donde el sordo se relaciona con personas que han tenido como primera lengua el español y no la lengua de señas colombiana.

15 A partir de los datos que arrojaron las entrevistas se advierten muchas otras fortalezas valiosas para la docencia, como el caso particular de la importancia de la investigación en el aula para la discapacidad. Dicha posibilidad no se abordó en este estudio, dado que se ponderó la necesidad de profundizar en las categorías expuestas. 
condición de discapacidad (primera categoría), lo cual conlleva conocer la normatividad que rige los derechos de la población con discapacidad, así como acompañar a sus estudiantes sordos en la reclamación de sus derechos. Por otro lado, el ser docente significa contar con un saber pedagógico que involucre la capacidad para comunicarse en lengua de señas. En otras palabras, se necesita un qué (enseñar) y un cómo (enseñarlo). Quizás uno de los valores del presente estudio es precisamente el querer encontrar el mínimo necesario con respecto al saber hacer del docente en el momento de abordar un trabajo con poblaciones en el campo de la discapacidad auditiva. Las dos categorías aquí presentadas son en realidad una base para el buen ejercicio de una labor docente; Lastimosamente, en muchos contextos de la sociedad colombiana dicho mínimo necesario no se cumple. Cabe destacar el hecho de que muchos de los actores de esta investigación se desempeñan en cargos que requieren liderazgo comunitario. De lo anterior se desprende que, en lo que concierne a este estudio, el éxito está más relacionado con la capacidad para influenciar a otras personas a través del trabajo con comunidades, lo cual trasciende el mero ideal del éxito económico (condiciones de vida con las cuales se cuenta), hacia una realización como profesional en términos de su relación con la sociedad.

Este estudio también brindó a un grupo de adultos, profesionales del campo de la educación, ${ }^{16}$ que presentan discapacidad auditiva la oportunidad de rememorar las vivencias por las que pasaron a lo largo de su formación académica. Hoy en día los maestros con quienes se realizó este estudio se desempeñan en diversos campos de la educación y la política, la mayoría son activistas docentes del campo de la discapacidad, al igual que sus maestros ya fallecidos. Reflexionar acerca de la labor docente de sus propios maestros implicó en cierta medida un ejercicio de autorreconocimiento, pero además — tal como se muestra en este estudio— posibilitó dar a conocer las bondades y no las falencias de los maestros que otrora dieron lugar a determinado acto pedagógico. La presente investigación permitió una multiplicidad de datos que en un futuro podrían dar lugar al dimensionamiento de nuevas categorías de análisis, ya que hay más variables que influencian el éxito profesional de los estudiantes sordos, entre ellas: 1) el modelo de escuela de sordos y 2) las competencias de los sordos en lectura y escritura en español. Existen también temas aún no abordados por el grupo de investigación, entre los que se cuentan: ¿Qué características detentan las familias en las que los hijos sordos alcanzaron el éxito profesional? ¿Qué causas motivan a los sordos a escoger las licenciaturas como principal

16 Algunos deseaban estudiar otras carreras, pero las universidades no les dieron otra opción que ser educadores. A esto se suma el hecho de que una vez son profesionales, a algunos casos no se les reconoce esta calidad, sino como trabajadores para modelo lingüístico. 
modelo de profesionalidad? Y, por último, ¿Cuáles son las principales causas de la deserción escolar de los estudiantes sordos en las universidades de Villavicencio (Meta)? Este tema no se abordó en este texto, y también se presenta con frecuencia en los claustros universitarios.

No obstante, el vincular las bondades de los maestros que en su momento bien hicieron en su ejercicio docente se debió al interés por contar la experiencia de quienes transforman a los sujetos a través de su práctica pedagógica, lo que da lugar a la reconciliación y la inclusión a través de su saber-hacer, propósitos que en Colombia aún están en proceso.

\section{Referencias}

Adajus. (2013). Discapacidad, justicia y Estado. Discriminación, estereotipos y toma de conciencia. Infojus Sistema Argentino de Información Jurídica.

Águila, C. (2010). Consideraciones acerca de la importancia del empleo del lenguaje no verbal para el exitoso desempeño profesional del jurista. IUs. Revista del Instituto de Ciencias Jurídicas de Puebla A.C. 25, 193-202.

Agurto, A. (2007). Las inteligencias múltiples en la educación para sordos. Polis [en línea], 17. http://journals.openedition.org/polis/4414

Álvarez, J. y Jurgenson, G. (2009). Cómo hacer investigación cualitativa. Fundamentos y metodología. Paidós.

Arendt, H. (2001). La condición humana. Paidós.

Bernstein, B. y Díaz, M. (1985). Hacia una teoría del discurso pedagógico. Revista Colombiana de Educación, 15. https://doi.org/10.17227/ 01203916.5120

Bolaños, J. y Pérez, M. (2019). Propuestas para la investigación cualitativa en educación artística. Educación y Educadores, 22(1), 51-63. 10.5294/edu.2019.22.1.3

Burad, V. (2010). Alteridad sorda. http://www.culturasorda.eu/resources/ Burad_Viviana_Alteridad_sorda_2010.pdf.

Carballeda, A., Diez, J. y Escudero, B. (Comps.) (2012). Cartografía social, investigación e intervención desde las ciencias sociales, métodos y experiencias de aplicación. Universidad de Patagonia.

Castellano, J. (2018). Relación entre indicadores de rendimiento y el éxito en el fútbol profesional. Revista Iberoamericana de Psicología del Ejercicio y el Deporte, 13(1), 41-49.

De Haro, J. y Castejón, J. (2014). Inteligencia emocional percibida, inteligencia general y éxito profesional en el inicio de la carrera: validez predictiva e incremental. Anales de Psicología, 30(2). http://dx.doi. org/10.6018/analesps.30.2.154621 
Dilthey, W. (2015). Introducción a las ciencias del espíritu. Fondo de Cultura Económica.

Fayad, J. (2015). Ciclos de vida como principio activo hacia una escolarización intercultural. Revista Colombiana de Educación, 69, 121-133.

Fernández, A. y Vasco, E. (2012). Dinámicas del reconocimiento en las narraciones de jóvenes con discapacidades. Revista Latinoamericana de Ciencias Sociales, Niñez y Juventud, 10(1), 467-479.

Ferreira, M. (2010). De la minus-valía a la diversidad funcional: un nuevo marco teórico-metodológico. Política y Sociedad, 47(1), 45-65.

Flick, U. (2015). El diseño de investigación cualitativa. Morata.

Foucault, M. (2003). Vigilar y castigar. Siglo xxı Editores.

García, M. (2005). La discapacidad hoy. Psychosocial Intervention, 14(3), 245-254.

Gardner, H. (2001). Estructuras de la mente. La teoría de las inteligencias múltiples. FCE.

Gardner, H. (2016). Las cinco mentes del futuro. Paidós.

Goffman, E. (2006). Estigma. La identidad deteriorada. Amorrortu.

González, O. (2019). La narrativa biográfica como una prometedora experiencia (auto)formativa en el trayecto de la formación docente. Revista Latinoamericana de Estudios Educativos, 15(1), 68-90.

Han, B. (2014). Psicopolítica. Neoliberalismo y nuevas técnicas de poder. Herder.

Henao, G. y García, M. (2009). Interacción familiar y desarrollo emocional en niños y niñas. Revista Latinoamericana de Ciencias Sociales Niñez y Juventud, 7(2), 785-802.

Huchim, D. y Reyes, R. (2013). La investigación biográfico-narrativa, una alternativa para el estudio de los docentes. Actualidades Investigativas en Educación [revista electrónica], 13(3), 1-27.

Laval, C. y Dardot, P. (2013). La nueva razón del mundo. Ensayo sobre la sociedad neoliberal. Gedisa.

Laval, C. y Dardot, P. (2015). Común. Ensayo sobre la revolución en el siglo xxı. Gedisa.

Llorent, V. y López, M. (2010). Atención educativa a la diversidad. Los alumnos sordos en las aulas de Portugal y Turquía. Foro de Educación, 8(12), 111-123.

López-González, M. y Llorent, V. (2013). ¿Deficiencia, discapacidad o identidad cultural? Interpretación de la sordera y respuestas en el sistema educativo en España. Revista Cefac, 15(6), 1664-1671.

Martínez, M., Mijares, B., Prieto, M., Ramos, L. y Díaz, B. (2012). Percepciones ante el éxito profesional: una filosofía de vida en estudiantes universitarios. Negotium, 7(21), 58-75. 
Mercé, O. (2012). Fama y éxito profesional en "Operación Triunfo" y "Fama ¡a bailar!". Comunicar, 39, 185-192.

Messina, G., Pieck, E. y Castañeda, E. (2008) Educación y trabajo. Lecciones desde la práctica innovadora en América Latina. Oficina Regional de Educación de la Unesco para América Latina y el Caribe Orealc/ Unesco Santiago.

Oliver, M. (1996). Understanding disability. From theory to practice. MacMillan.

Ortiz, A. (2017). Configuración epistémica de la pedagogía. Tendencias que han proliferado en la historia de la educación. Historia de la Educación Latinoamericana, 19(29), 165-195.

Palacios, A. y Romañach, J. (2008). El modelo de la diversidad: una nueva visión de la bioética desde la perspectiva de las personas con diversidad funcional (discapacidad). Intersticios. Revista Sociológica de Pensamiento Crítico, 2(2), 37-47.

Pasek, E. (2008, febrero). La construcción del problema de investigación y su discurso [ponencia]. Universidad Fermín Toro. Cabudare, Venezuela.

Pérez, O. (2014). Un dilema sobre la minoría sorda. Revista de Bioética y Derecho. 30, 125-136.

Reyes, E. y Colás, I. (2017). El activismo social y sus particularidades en la educación. EduSol.

Robles, B. (2011). La entrevista en profundidad: una técnica útil dentro del campo antropofísico. Cuicuilco, 18(52), 39-49.

Rodríguez, N. García, D. y Jutinico, M. (2008). Inclusión de estudiantes sordos a la vida universitaria: una experiencia significativa en la Universidad Pedagógica Nacional. Revista Colombiana de Educación, 54, 170-195.

Saldarriaga, O. (2002). El oficio de maestro, saber pedagógico y prácticas culturales en Colombia, 1870-2002. Memoria y Sociedad, 6(12), 121-149.

Santos, D., Baquero, S. y Beltrán, M. (2008). Adaptaciones pedagógicas para estudiantes sordos en la Universidad Nacional de Colombia: reflexiones curriculares. Revista Colombiana de Educación, 54, 120-141.

Unda, M. y Gutiérrez, A. (2015). Viajes y encuentros pedagógicos como experiencias de formación de maestros. Nodos y Nudos, 4(39), 7-26.

Vélez, I., Rátiva, S. y Varela, D. (2012). Cartografía social como metodología participativa y colaborativa de investigación en el territorio afrodescendiente de la cuenca alta del río Cauca. Revista Colombiana de Geografía, 21(2), 59-73.

Zuluaga, O. (1999). Pedagogía e historia. La historicidad de la pedagogía: la enseñanza, un objeto de saber. Editorial Universidad de Antioquia, Anthropos, Siglo del Hombre Editores. 